\title{
Case Report \\ Joubert Syndrome Diagnosed at 16+6 Weeks Gestation and Molar Tooth Sign by 3D Modality
}

Prabha Sinha, Shabnum Sibtain

\begin{abstract}
- - - - - - - - - - - - - - - - - - - - - - - - - - - - - - -
ABSTRACT:

Joubert's syndrome is a rare genetic disorder. It is an autosomal recessive neuro-developmental disorder involving mid and hind brain. This report describes a fetus at gestational age 16weeks+ 6 days, who presented with characteristic Molar tooth sign on the antenatal scan. This sign is a salient feature of Joubert syndrome (JS). Mostly the diagnosis of JS is made after birth. Few cases are diagnosed prenatally by ultrasound.
\end{abstract}

Key words: Joubert syndrome, mortality, genetic disorder, brain malformation

How to cite this Article:

Sinha P, Sibtain S. Joubert Syndrome Diagnosed at 16+6 Weeks Gestation and Molar Tooth Sign by 3d Modality. J Bahria Uni Med Dental Coll. 2020;10(3): 249-50 DOI: https://doi.org/10.51985/JBUMDC2020024

\section{INTRODUCTION:}

Joubert syndrome (JS) is a rare autosomal recessive genetic disorder. ${ }^{1}$ It affects the area of brain involved in balance and coordination. It is clinically heterogeneous associated with retinitis pigmentosa and multiorgan involvement. JS affects 1 in 80,000-100,000 newborns.$^{2,3}$ It is likely to be underdiagnosed. Variety of genes disorder cause Joubert's syndrome. It is commonly found in Arab, Jewish, French, Canadian, and Netherland populations. It is autosomal recessive but it can rarely be inherited as an X-linked recessive pattern. The recurrence risk is $25 \%$ in most families. ${ }^{4}$ Joubert syndrome is primarily nervous system disorder with cerebellar vermis hypoplasia or absence. There is malformation of brain stem. This leads to defect in axon signals. In addition to neurological symptoms there may be hypoplastic phallus, renal cysts, cleft lip or palate, polydactyly and tongue abnormalities. Prenatal sonographic findings are relatively nonspecific. The molar tooth sign is observed on 3D ultrasound or MRI scan; and is a salient feature of this disease.

Case: A 28-year-old, G8 P5+2 had consanguineous marriage. History was significant for gestational diabetes, controlled on diet. She has four healthy children and one child with Vermis Agenesis and Polyhydramnios. The family history was noteworthy for recurrent fetal loss with hydrocephalus.

She was referred to Fetal Medicine Unit for early anomaly scan at 16 weeks +6 days due to previous history. On ultrasonography scan; characteristic molar tooth sign, absent

Prabha Sinha
Consultant Tawam Hospital
Abu Dhabi, UAE.
Shabnum Sibtain
Assistant Professor
Azra Naheed Medical College, Lahore, Pakistan
Email: s_sibtain@ hotmail.com
Received: 06-Apr-2020
Accepted: 25-Jun-2020

cerebellum and vermis, frontal bossing with hypertelorism, dilated anterior horn of the lateral ventricle, enlarged cisterna magna with posterior encephalocele, kyphoscoliosis and bilateral renal pelvic dilatation were observed. The diagnosis of Joubert syndrome was made based on these findings and the history of previously affected child. The couple was counselled about the findings and outcome. Parents decided for termination of pregnancy at 17 weeks of gestation. Sample from aborted fetus was consistent with the diagnosis of Joubert syndrome type 21. Genome sequencing confirmed CSPP1 (NM_024790.6; exon 17,) homozygous likely pathogenic variant c.2131_2132del p. (Ser711Leufs*11)

\section{DISCUSSION:}

Joubert syndrome a genetically heterogenous disorder was first described in 1968. ${ }^{5}$ The disorder is characterized by molar tooth $\operatorname{sign}^{6}$ due to thickened cerebellar peduncles. A cleft is visualized between the cerebellum. The sign can be visualized on a 3D ultrasonography scan or MRI scan. This syndrome is a result of malformation of the brain. The diagnosis of this condition is based on the physical symptoms and radiological findings, and confirmed by testing for specific genetic mutations. The symptoms of the disease present in very early childhood, primarily involving neuromuscular system along with developmental delay. Other associations are disorders of kidney, liver and eyes. Mutation of 21 genes has been observed in relation to JS. ${ }^{7}$ Early prenatal genetic testing is advised for at risk couples. Fetal brain imaging can provide useful information in the last trimester. ${ }^{4,8}$

The management of this syndrome is multidisciplinary. The neonates have respiratory and feeding difficulties and these problems need to be addressed as priority. Cognitive behavioral therapy is required in young patients for neuropsychological support and rehabilitation.

The prognosis of JS varies and is dependent on the extent and severity of organ involvement. Mostly the diagnosis of JS is made after birth. Few cases are diagnosed prenatally by ultrasound. The diagnosis is usually missed because of 
nonspecific features that can occur in Dandy-Walker malformation and other cranial syndromes. ${ }^{9}$ The ultrasound is the primary screening method to detect posterior fossa abnormalities and diagnosis of JS is confirmed on MRI. ${ }^{10}$ The ultra-sonographer may just observe the nonspecific features due to lack of knowledge and rarity of this syndrome.

There is lack of enormous data on the long-term outcome of children affected by JS. Children with associated complications have poorer outcome. The sonographic findings in fetuses with JS are relatively nonspecific in the prenatal period. In this case molar tooth sign was identified at $16+6$ weeks in $3 \mathrm{D}$ scan. The pregnancy was terminated at 17 weeks because of poor outcome. Early diagnosis is of great value in Islamic country as termination of a pregnancy is not feasible beyond 18 weeks gestation. Previous history with ultrasound findings helped to formulate early diagnosis. Careful history is important to assess risks of JS and optimal management is multidisciplinary. Our findings of JS in early stages are mostly the same as found in literature to date. The risk of recurrence is $25 \%$ in most families. ${ }^{11}$

\section{CONCLUSION:}

Early prenatal detection of JS can lead to early termination of pregnancy. This can reduce the emotional and financial stress on the couple. These prenatal sonographies can also

Figure 1: Molar Tooth sign on Ultra-sonography scan

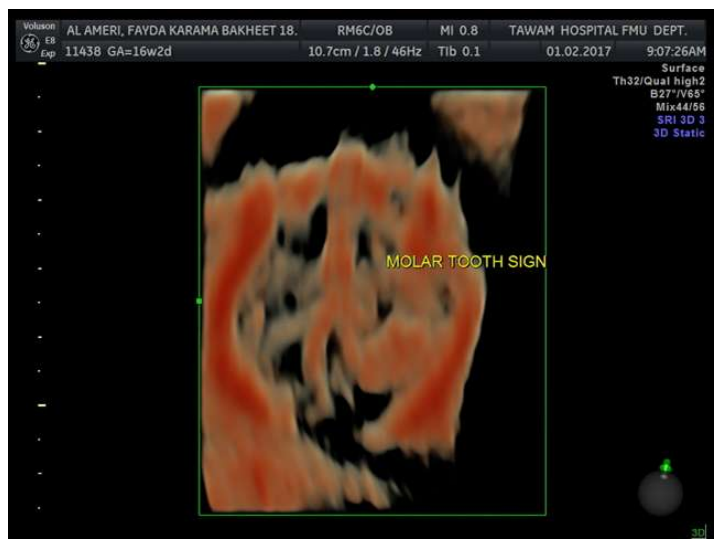

Figure 2: Fetal Ultrasound at $16+6$ weeks Gestational age showing agenesis of vermis.

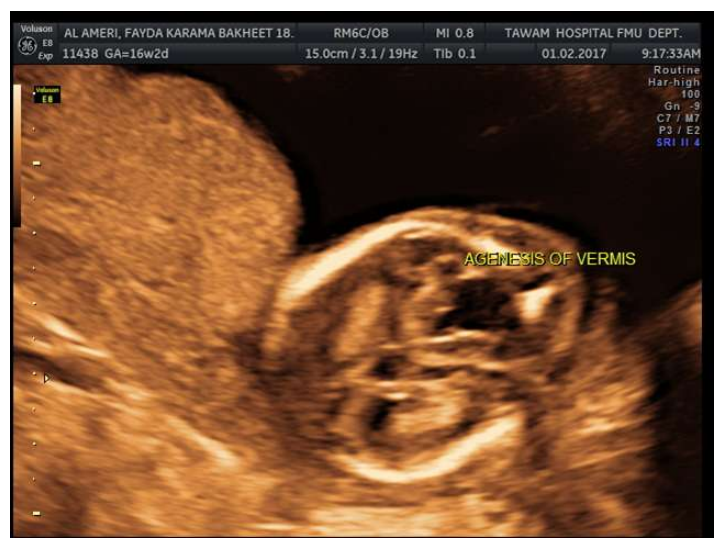

help the differentiate mid and hind brain abnormalities. Therefore, a protocol to monitor the pregnancies at risk of JS should be followed. It can be achieved by serial ultrasonography performed by a specialist followed by MRI. The prenatal genetic analysis of couple and fetus is useful in making a definitive diagnosis. The affected couple should be counselled in this regard. JS is associated with high mortality, identifying the cause and time of death will help in development of guidelines to improve the outcome of JS.

Author Contribution:

Prabha Sinha: Substantial contributions to the conception or design of the work; or the acquisition, analysis, or interpretation I of data for the work

| Shabnum Sibtain: Drafting the work or revising it critically | for important intellectual content

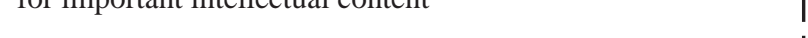

\section{REFERENCE:}

1. Dempsey JC, Phelps IG, Bachmann-Gagescu R, Glass IA, Tully HM, Doherty D. Mortality in Joubert syndrome. Am J Med Genet A. 2017 May;173(5):1237-1242. doi: 10.1002/ajmg.a.38158. Epub 2017 Mar 28. PMID: 28371402

2. Parisi MA, Doherty D, Chance PF, Glass IA. Joubert syndrome (and related disorders). Eur J Hum Genet. 2007 May; 15(5):511-21.

3. Kroes HY, van Zon PH, Fransen van de Putte D, Nelen MR, Nievelstein RJ, Wittebol-Post D, van Nieuwenhuizen O, Mancini GM, van der Knaap MS, Kwee ML, Maas SM, Cobben JM, De Nef JE, Lindhout D, Sinke RJ DNA analysis of AHI1, NPHP1 and CYCLIN D1 in Joubert syndrome patients from the Netherlands. Eur J Med Genet. 2008 JanFeb; 51(1):24-34.

4. Brancati F, Dallapiccola B, Valente EM. Joubert Syndrome and related disorders. Orphanet J Rare Dis. 2010 Jul 8;5:20. doi: 10.1186/1750-1172-5-20

5. 5)Doherty D. Joubert syndrome: insights into brain development, cilium biology, and complex disease. Semin Pediatr Neurol. 2009 Sep; 16(3): 143-154

6. Juric-Sekhar G, Adkins J, Doherty D, Hevner RF, Joubert syndrome: brain and spinal cord malformations in genotyped cases and implications for neurodevelopmental functions of primary cilia. Acta Neuropathol. 2012 May;123(5):695-709. doi: 10.1007/s00401-012-0951-2. Epub 2012 Feb 14

7. Romani M, Micalizzi A, Valente EM. Joubert syndrome: congenital cerebellar ataxia with the molar tooth. Lancet Neurol. 2013 Sep;12(9):894-905. doi: 10.1016/S14744422(13)70136-4.

8. Valente EM, Dallapiccola B, Bertini E, Joubert syndrome and related disorders. Handb Clin Neurol. 2013;113:1879-88. doi: 10.1016/B978-0-444-59565-2.00058-7.

9. Lingling Z , Limei XPrenatal Diagnosis of Joubert Syndrome: A Case Report and Literature ReviewMedicine (Baltimore) 2017 Dec; 96(51):e8626

10. Jingjing X, Lili Z, Wei J,Qin Z, Ting W and Hong Li Biomed Res Int. 2018 may; Published online 2018 May 31.

11. Baris B, Emre, Goksun I, Semiha C, and Hatice A Diagnosis of Joubert Syndrome via Ultrasonography J Med Ultrason (2001)2017 Apr; 44(2):197-202 